\title{
Electronic Leadership in Time of Crisis: Challenges and Opportunities in the Light of COVID-19 Quarantine
}

\author{
Faiza Adil Gonaim \\ Department of Administration and Fundamental of Education, University of Jeddah, Jeddah, Saudi Arabia
}

Email address:

fghonaim@uj.edu.sa, fgonim@yahoo.com

\section{To cite this article:}

Faiza Adil Gonaim. Electronic Leadership in Time of Crisis: Challenges and Opportunities in the Light of COVID-19 Quarantine. International Journal of Education, Culture and Society. Vol. 6, No. 2, 2021, pp. 54-62. doi: 10.11648/j.ijecs.20210602.13

Received: April 4, 2021; Accepted: April 16, 2021; Published: April 26, 2021

\begin{abstract}
E-leadership can be seen from different lenses, considering the nature multidisciplinary. It can be seen from the lens of computer science, education, management, information system. Be that as it may, right now E-leadership has been drawn closer from the focal point of educational leadership. The disarray that was brought about by the spread of coronavirus has affected everyone's life. The educational segments have been terminating to keep up the progression of the instructive procedure. Similarly, the weight on educational leaders has raised fundamentally. The examination in e-leadership is as yet constrained. In this manner, more examinations are expected to augment the impact of e-leadership extraordinarily in times of emergency which force virtual correspondence and accomplishment. As educational leaders incorporate impacting and creating others, they are relied upon to proceed with their job normally. Educational leaders experience difficulties in playing out their job electronically in emergency time. In any case, as the emergency causes the introduction of chances, leading electronically in the touchy time COVID 19 isolate can mean different open doors for pioneers and instructive improvement. This examination intended to explore the opportunities and difficulties of e-leadership in times of emergency, especially in COVID 19 isolate. The examination was held utilizing qualitative request in which a top to the bottom meeting was led with educational leaders to investigate the difficulties and the potential chances of leading electronically. The discoveries are relied upon to urge educational leaders to make better move and collaboration in leading electronically uncommonly in times of emergency. Moreover, it helps approach producers to give the required readiness to educational leaders to lead adequately in various situations. Moreover, the investigation can add an adequate foundation for proficient e-instructive leadership.
\end{abstract}

Keywords: E-leadership, Leadership in Crisis, Covid-19 Leadership, E-leadership Challenges, E-leadership Opportunities

\section{Introduction}

The explosion of information and technology force countries and people to change their lifestyles. This era characterized as the age of information. The role of leaders is vital as they are the fuel that accelerates the achievement of goals. Although leadership is a contested concept that has no consensus in its definition, it is highly known as a social influence process [13]. It is also a significant factor for the success and the effectiveness of organizations [29]. The value of this phenomenon is developing a supportive environment, shared responsibility, reciprocal caring and nurturing sustainability, and competency of the organizations. Living in the age of information imposes leadership to be performed in wider platforms that are leading in a virtual context. Avolio and Kahai asserted that under this form of leadership, a fundamental change of leadership is required [2]. This new model of e-leadership is young; hence, it can be called an emergent leadership [10]. What is expected from leaders in a real context such as influencing to achieve goals can be performed in a virtual context.

However, the nature of virtual leaders-team interaction imposes roles and techniques which are different from the traditional face to face leadership as Garcia asserted after his literature review in the subject [15]. In practicing e-leadership, leaders can be communicating with people who already have met face to face or with whom they don't know or have met before. The understanding of mediating leadership in a virtual context is still limited [10]. The complexity of leadership which comes from the various leaders' responsibilities such as creating a vision, reinforcing values, motivating team members and managing change add hock on leaders when it is 
expected to be done electronically. Research shows that leading digitally requires greater effort [10]. Therefore, considering the expansion of the use of technology and the vitality of leadership especially in times of crisis, it is imperative to understand how technology impacts leadership and how leadership is impacted by technology. As the Coronavirus disease (COVID-19) pandemic and its impacts have affected the entire world, it became a fruitful platform for studying e-leadership in crisis to investigate the opportunities and challenges that educational encounters throughout the isolation of COVID-19 crisis.

\section{Problem Statement}

The quick-changing universe has time-pressed all segments broadly and universally. The cutting-edge innovation and digitalization forced different changes that require diverse methodology of interchanges and connecting. It has been hard to discover instructive settings without the commitment to innovation. In this manner, it is significant for leaders to create satisfactory comprehension of the proper online correspondence and the aware conduct. Although digitalization has been penetrating every sector, investigating e-leadership in education showed that research in this subject still limited $[1 ; 31 ; 35]$. Akram and Khan also asserted the lack of literature that addresses the e-leadership framework or model for effective practices [1].

Further, this comprehension of e-leadership is essential when leading on the web in times of emergency. An emergency can take diverse structures and sizes to rely upon the unique circumstance and its status for experiencing them. These emergencies that experience leaders make strain and dubiousness for the job of administration as Mazánek [26] stated. The absence of understanding the job of e-leadership in times of emergency can be a reason for frustrating arrangements or in any event, extending the emergency because of miscommunication or error. By surveying the writing in e-leadership, there is a need that the writing addresses leading in online conditions. Besides, after narrowing the inquiry down to explore online leadership in times of emergency, the writing gives little direction in the subject.

\section{Objectives}

To research educational leaders' point of view of leading electronically in the isolated time which brought about by COVID-19. Also to explain the open doors that connate in leading electronically in time of the emergency.

To comprehend the difficulties that educational leaders experience all through their experience of e-leadership in the hour of the emergency.

\section{Utilization}

For a functioning viable initiative to work as a basic unit in the instructive foundation, leaders must show a viable administration in conveying legitimately or electronically. Particularly in the present eccentric changes or emergency which are globalization, and the development innovation are their causes [18]. Notwithstanding, a successful authority in direct circumstances may not work in a virtual condition. In addition, in times of emergency, authority practices and demeanor become indispensable for forestalling the exacerbation of the emergency. Subsequently, examining e-leadership practices in times of emergency is a productive zone of research. Since innovation have been involving a basic piece of the present correspondence and interaction, this investigation of e-leadership in time of emergency can conceivably upgrade positions of leadership in leading in various conditions. Simultaneously, asking into the difficulties that educational leaders experience in leading electronically in time of emergency could add to the improvement of various, compelling practices to adapt to the difficulties. Additionally, exploring the open doors in driving in such cases can be intriguing for extricating openings from challenges.

The vagueness and unpredictability of leading electronically in time of emergency expand the weight on the educational leaders who must deal with these overwhelming obligations. However minimal observational research has explored e-leadership rehearses that help educational leaders to stand the perplexing duties of leading electronically in time of emergency. Henceforth, this examination is a commitment to the writing as it delineates e-leadership practices for instruction which can turn into a guide for others involving that job of educational leaders.

Research needs to fill in as an impetus for approach creators to execute positive change [28]. The discoveries of this examination could propel arrangement producers in instruction to structure leadership planning programs that incorporate e-leadership successful practices. Such projects would help current educational leaders and get ready for those who are a potential possibility for the essential situation of e-leadership, so they can contribute beneficially to their association.

The last advantage of this investigation is that it directed qualitatively. The vast majority of the information gathered about instructive leadership in Saudi Arabia are quantitative. For example, Smith and Abouammoh [32] called attention to that is to produce unique bits of knowledge concerning the improvement of advanced education, it would be worth served by utilizing subjective techniques.

As an educational leaders who is leading electronically in the hour of emergency COVID 19 isolate, I am particularly situated to assemble data from members who involve comparative position and can decipher discoveries with regards to the way of life e-leadership in time of emergency.

\section{Background for the Study}

\section{E-Leadership}

Over the last two decades, the concept of e-leadership has been evolving with considerable attention to the phenomena $[17 ; 25]$. The concept is overlapped with other concepts such as technology/ IT/ technology/ online leadership. There are different definitions for e-leadership; yet, the most useful definition is presented by Avolio and colleagues [3]. They are considered pioneers in bespoke e-leadership as a concept that explores the effect of AIT (Advanced Information Technology) on leadership. They defined it as a social influence process embedded in both proximal and distal contexts mediated by AIT that can produce a change in attitudes, feelings, thinking, 
behavior, and performance [4 p. 107]. In another articulation, it also defined as a "social influence process mediated by Advanced Information Technology (AIT) to produce a change in feelings, thinking, behavior, and/or performance with individuals, groups, and/or organizations" [3]

The purpose of e-leadership is to maintain the relationship of the members in an organization enhanced by the structure of the organization [2]. Further, it incorporates the dissemination of information and instruction digitally. All of which can be inspiring using an electronic effective leadership approach. In his micro-level analysis of instant message conversation, Darics provided strategies from real-life communication and nonverbal communication that can enhance leaders' practices of leading virtually [10]. Furthermore, the literature emphasized that better awareness of the use of digital media makes effective use of technology which contributes to effective leadership [22].

Not only the explosion of technology increased pressure on leaders to pay more attention to e-leadership, but other factors are also involved. For instance, access to information has not been exclusive to leaders only, also the followers can attain information which increases the pressure on leaders to quickly answer questions and justify their decision. In addition, it has been easier reaching out to the leaders, and communication has been more indelible than before [2].

In an attempt to identify the best practices for e-leadership, Malhotr, Majchrzak and Rosen investigated the best leadership practices for leading virtually by studying virtual teams [25]. They found the practices are: (a) reinforcing trust; (b) fair distribution for diversity with appreciation; (c) effective monitoring for the work and the team; (d) extending the visibility of virtual members; (e) ensuring the individual benefit from the team. Brake suggested using practical guidelines for effective e-leadership [7]. It includes applying cultural intelligence, precise communication, proactive thinking, and predictability. Plenty of literature emphasized the importance of skills that are related to communication, motivation, problem-solving, and conflict management.

E-leadership incorporates numerous advantages. For instance, creating a more adaptable organization than a traditional organization, creating team members considering specialization regardless of geographical existence, the flexibility in work performance, availability of communication [35]. Although e-leadership has great benefits in the organization, yet it has some drawbacks. It added the pressure on leaders to be present which increased the leading time. Unethical leaders can affect a wide range of audiences. Some of the challenges are demonstrated in the difficulty of reaching a higher level of performance because of inefficient interaction [35]. They also pointed out that the process loss is another difficulty that can be caused by a lack of shared understanding, mistrust, or low cohesion. In his literature review on e-leadership DasGupta pointed to the challenges of bridging physical distances, conveying enthusiasm, communicating effectively, and building trust [11]. Isolation and confusion are other challenges that can be avoided by practicing practical guideline [7].

\section{Leadership in Crisis}

A crisis can take different types, forms, and sizes [18]. The causes of the crisis are varied, they could be external causes such as the spread of coronavirus or internal causes such as a wrong decision. Communication in crisis become a sensitive issue as the panic cause misunderstanding, rumors, and fake news [5]. For this reason, Ulmer pointed out that the field of communication in crisis has to be normative science not only a positive science to improve crisis communication [33]. Therefore, the competency of the leaders plays a significant role in times of crisis.

Bhaduri in his study of crisis management deduced that four types of leadership are required in crisis: transactional, transformational, cognitive, and directive leadership [6]. Organizational crisis management process is explained through the five-stage life cycle, including signal detection, prevention, damage containment, recovery, and learning. Four types of leadership are included; directive, which is critical during crisis management. Mazánek asserted that in times of crisis, authoritative leadership is typical in times of crisis as people under the threat expect leaders to centralize authority [26]. Jones and Chauhan study found the effective leadership in crisis required some traits such as positive attitudes, calm conduct, confidence, creativity, and resilience [18]. According to King team effectiveness is crucial in times of crisis [20]. Their effectiveness resulted from team composition, leadership efficiency, organizational culture, and task knowledge.

Although the crisis appears as an unfavorable occurrence in an organization, it can be a cause for development. For instance, Probert \& James argued that the ambiguity and uncertainty associated with a crisis can be an opportunity for leadership development [30]. To clarify, the crisis provides an ideal event for fostering by recognizing what needs alteration. Kouzes and Posner asserted that the challenges that encounter leadership are assets for more improvement [21].

Mazánek in his study on the role of leaders of effective management in times of crisis identified the importance of crisis communication, leaders' behaviors, and their leading style and reinforcing team effectiveness [26]. Kielkowski also pointed out that successful leadership in a crisis is developed before the occurrence of emergencies such as building team effectiveness and effective communication plan [19]. Exercising challenges and examining team responses assist in confronting real challenges. It allows building confidence and demonstrates that recovery is possible [19]. "Calmness in the eye of the storm" which is articulated by Lucy is a crucial strategy in challenging time as it promotes team members' confidence and belief in passing the calamity [24].

\section{COVID-19 Crisis}

The pandemic of coronavirus (COVID-19) is a massive health crisis that affected worldwide. It is "an infectious disease caused by a newly discovered coronavirus" [34]. The spread of the disease is massive causing deaths and affecting various sectors. At the time of conducting this research, there is no specific vaccines or treatment for it, yet the ongoing trial for potential treatment is unstoppable (World Health 
Organization). Bavel, et al. found that as the virus has affected all life aspects of the people, the response has to include social and behavioral science [5]. In their article of the response to the pandemic, they asserted that leadership among the crucial factor in responding to the pandemic. Leadership influences people on responding and promoting cooperative behaviors. Furthermore, leaders are expected to solve immediate problems as well as improving the approach of responding to crises in general [16].

\section{Research Design}

Looking into and evaluating written works in the region of research offers a way for the specialist to pick the proper techniques [23]. In qualitative research as in all exploration, testing implies choosing the individuals who take part in the study to gain information that applies to the examination questions [23 p. 101]. The way toward choosing members must guarantee choosing individuals who can give pertinent data. Creswell brought up that an analyst could utilize more than one methodology for examining in an examination [9]. At first, I utilized the advantageous and deliberate testing method, otherwise called nonprobability inspecting [23]. Intentional examination implies that the "inquirer chooses people and destinations for study since they can deliberately educate a comprehension regarding the examination issue and focal wonder in the investigation." [9 p. 156]. I utilized the snowballing strategy to choose leaders who are mindful and fair. An email was sent to the recognized members, with a clarification of the motivation behind the investigation, to welcome them to take an interest in the examination and to answer to my email in the event that they acknowledged the greeting.

A subjective report was directed including intentionally inspecting leaders in the instructive area who have experience leading electronically in the time of COVID-19 isolate. The utilization of a semi-organized meeting allowed me to complete the examination. The use of qualitative approach helped to achieve the member point see with profound understanding, not at all like the utilization of quantitative research. Guba and Lincoln expressed, "subjective information, it is stated, can give rich knowledge into human conduct" [12 p. 106]. English underlined that understanding leadership practice is far from straightforward math practices or results [14]. Merriam pointed out that qualitative researchers develop with an enthusiasm understanding of "how individuals comprehend their life and their experience" [27 p. 38]. In this manner, the crucial trait of qualitative research is that people who connect with their social universes develop reality. Further, analysts are special to get as close as conceivable to the member to get familiar with their emotional experience regarding the inquiries posed [9].

The information analyzed intently, and each line or portion was given a name. Transcripts were re-perused a few times to guarantee steady information naming practices. This methodology keeps the scientist open to any conceivable hypothetical heading, brings the specialist into more profound comprehension, and permits the analyst to see territories wherein more information is required [8]. Coding is the primary systematic advance, which helps to move from names to a more elevated level of deliberation. After having all the information coded, the following period of coding, which is engaged in coding, was utilized. After that, related classifications were chosen and put under focal topics.

\section{Findings}

The research aims to investigate educational leaders' point of view of leading electronically in the isolated time which brought about by COVID-19. To accomplish this goal, the focus was to answer two questions:

What are the open doors that connate in leading electronically in time of the emergency?

What are the difficulties that educational leaders experience all through their experience of e-leadership in the hour of the emergency?

By using the word frequency table of NVIVO numbers of themes emerges regarding the research questions. The themes resulted from the coding which was clustered under an appropriate category. For the first question, four themes emerged after. Whereas, for the second question, the participants pointed to varieties of opportunities which are emerged in driving electronically. These opportunities are characterized under four categories: cognitive aspects, professional aspects, organizational structure aspects, and personal aspects (Table 1).

Intellectual Aspects

The first category, intellectual aspects, contains three themes. The first theme is that obtaining varieties of courses and programs. The participants asserted that leading electronically has opened the door to rich intellectual obtainment. Abundant of courses and programs were offered for leaders to nourish the best practices of management during the crisis. The courses and programs did not focus on managing work performance, it also includes soft skills that suit interactions in times of crisis. Furthermore, intellectual development as the participants pointed it expanded to include teachers, students, and parents. They clarified that reaching out to parents was not an easy task during the regular life routine, yet under the quarantine of COVID-19, the digital connection has increased due to the follow up of the parents to their children.

Providing varieties of courses and programs is another opportunity that has strengthened in the crisis. Educational leaders found that the crisis is an opportunity to reach out electronically to wider society. Such as parents, society, and stakeholders besides the teachers and students. The side effect of the crisis, quarantine, increased the feeling of guilt of many people; hence, they turned out to enrolling in a variety of courses and programs to reduce the sense of guilt of missing out.

Benefiting from global experiences. The participants pointed out that the COVID-19 crisis opens the door to benefit from global experiences in leadership. For instances, they 
pointed to the advantages they attained from leaders who share their experiences of leading online; besides, the number of organization which has provided free access to valuable programs such as Harvard School.

Professional Aspects

The participating educational leaders pointed to the number of openings that are related to the profession. Enhancing the technical shift is an essential opportunity. One of the participants articulated that, "COVID-19 is the strongest motive for a technological shift in education". One of the leaders' goals is to promote the shift into technology especially in the era of technology explosion. The shift was intended to be involved gradually, yet the crisis pushed this shift in such a short time. For instance, virtual classes, meetings, thesis defenses have been the media for the accomplishment of educational works. All of which have opened up technological thinking and acceptability. Before the crisis, there were many people against the reliance on technology for the lack of expertise in using technology or the fear of using it. For instance, the opposition sometimes comes from old fashioned teachers, parents, or even students. However, the real experience of dealings with technology has broken all the fear and has proven its practicality.

Enhancing professional skills is another opportunity that emerged out of leading electronically. The participants have pointed to numbers of professional skills which have been reinforced by leading electronically. One of which is time management skills. Participant T. W. Pointed, "leading electronically has helped me to prioritize my tasks and manage my time based on the priorities". Other participants have emphasized that their skills in working under pressure have strengthened. G. M. articulated, "People expect leaders to be on call $7 / 24$, which increases the demand on leaders, yet the experience has enhanced my skills to fulfill works even under the high demands". In addition, the planning skills have improved to accomplish tasks that are required to be performed at a particular time such as meetings for leaders or teachers or exams for students.

The emergence of new job opportunities is also an opening that came out of electronic leadership in crisis. For instance, the participant pointed out that in time of crisis, workers are needs to feel safe and things are under control. For this, organizations necessitate psychological experts to alleviate stress and uncertainty feelings. Another job opportunity is related to digital facilitation workers such as programmers and trainers.

Working digitally increase job satisfaction as the participants pointed. Leaders have earned more time in quarantine as they have saved the commute time by leading electronically. Women have found it more practical to lead and work from home as they could manage works along with household responsibilities.

\section{Organizational Structure Aspects}

Leading electronically in the COVID-19 crisis created and reinforced several factors related to organization structures as the participants indicated. Improving the means of communication is one of the opportunities. Before the pandemic, it was the dominant belief is that direct communication in running work such as meeting is the only productive approach. Yet, after experiencing leading work online, it is proven that running work, meetings, can be done effectively using different mediums such as blackboard, teams...etc. The feature of this approach of communicating is the flexibility in communicating at any time; besides, the smoothness in following up. Furthermore, these approaches to communicating openly the door to increasing the number of accommodated learners.

Reframing organizational structure is another mentioned opportunity related to organizational structure. Leading electronically allowed leaders to detect limitations and vulnerability of organizational structure. For instance, M. N stated, "We found that many of the work procedures were taken long time be achieved because of meaningless bureaucracy and centralization!". Thus, organizational structure after the experience of e-leadership in the crisis can be rethought to be more practical with less centralization.

In addition to reframing team members to be equipped in leading their duties during an emergency; this considers an early preparation for crisis. The early preparation for crisis as the participants indicated includes; analyzing the past, the present, and foresight the future. Analyzing the past provides lessons whereas, analyzing the present while foresight the future assets in detecting signs of crisis and how should they perceived. Also, allocating the budget for confronting crises is early preparation for crisis.

\section{Personal aspects}

The participants found that leading electronically in the crisis of COVID-19 has implanted and developed various personal skills such as increased self-reliance, creativity and innovation development, and crisis skills management. For the first opening, increased self-reliance, leading electronically gave leaders chances to be independent in managing works. Unlike leading face to face which restricts leaders' freedom in management.

Furthermore, it gave leaders opportunities to think creatively and innovatively in managing work as the sense of delegation increased. For instance, M. A. indicated, "leading online grown in our creativity to perform tasks the best way they can be done". W. O. added, "Leading online reduced boredom which was associated with the quarantine as leaders think to come up with new ideas in running work". For example, educational leaders created motivational approaches such as providing free courses, competition, research awards. All of which contribute to the developing educational sector.

Leading from a distance in a time of crisis is not free from surprises. Thus, leaders' crisis management skills are reinforced. For illustration, they find a way to deal with unpredicted difficulties. Their patience increased accordingly, especially with the inevitable defect of technology. In addition, their skills of adaptation improved as well. As the ripple effect of the crisis is varied, people become uncertain with unstable feelings. Thus, they become in need of calming and optimistic leaders. Therefore, leaders found that their calming and facilitating skills have flourished. 
Table 1. The open doors aspects that connate in leading electronically in time of the emergency.

\begin{tabular}{ll}
\hline Categories & Themes \\
\hline \multirow{3}{*}{ Intellectual Aspects } & Obtaining varieties of courses and programs \\
& Providing varieties of courses and programs \\
& Benefiting from global experiences \\
Professional Aspects & Enhancing the technical shift \\
& Enhancing professional skills \\
& The emergence of new job opportunities \\
Organizational Structure Aspects & Increased job satisfaction \\
& Improving means of communication \\
& Reframing organizational structure \\
Personal Aspects & Early preparation for crisis \\
& Increased self-reliance \\
& Creativity and innovation development \\
\hline
\end{tabular}

The challenges of e-leadership in the crisis of COVID-19

Although there are valuable opportunities for leading electronically that emerged out of leading in the crisis of COVID-19, the experience has several challenges. In answering the second question, which is: What are the difficulties that educational leaders experience all through their experience of e-leadership in the hour of the emergency? The participants pointed to the number of challenges. After analyzing their responses, the challenges fall under two categories: internal management challenges and external management challenges (Table 2).

Internal management challenges

The magnitude of workload has increased causing internal management challenges for educational leaders. They were expected to be on call 24/7. Thus, this caused irritation and form a challenge for leaders to make a balance between their personal demand and work demands. N. T. stated, "Working from home, made some people think that you have to work and respond the whole day, without respecting personal time!"

In addition, leaders encountered difficulty in practicing the whole essence of leadership, which is influence, electronically. Using body language contributes significantly to influencing others; thus, educational leaders believe that leading electronically deprives them of the impact of body language. Having said that, the culture might hinder the full use of technology which allow expressing body language via face showing online program. For instance, some people believe that opening a camera in an online meeting interferes with their privacy.

The difficulty of estimating the level of people interact with online leadership forming another internal challenge for leaders. The distance and the openwork from home hider discipline which eases the process of evaluation. This causes anxiety for leaders to ensure the workflow. Besides, they become also anxious for the sake of providing an equitable learning opportunity for all.

\section{External management challenges}

Educational leaders have encountered external challenges concerning the practicality of technology and people interact with it. N. T. articulated, "Even technology is a great breakthrough, yet in many times it portrayed us with unexpected defect". Not only the defect of technology form challenges but also the lack of understanding of using it or the lack of the availability of it with every beneficiary. Accordingly, many refuses to interact with leaders when they being led electronically. For instance, the sudden transfer of education from classrooms to virtual class, many students, and teachers could not accept and interact effectively with this transformation. In addition to the lack of self-discipline of the educational beneficiary added to the challenge that leaders have encountered.

Table 2. The challenges of e-leadership in the crisis of COVID-19.

\begin{tabular}{ll}
\hline Categories & The challenges \\
\hline & Balancing personal life and work demands. \\
Internal management challenges & Lack of practicing full leadership/body languages. \\
& Cultural differences. \\
& Realizing the extent of people interaction. \\
& Practicality of technology. \\
External management challenges & People interaction. \\
& Lack of self-discipline. \\
& Personalities and cognition differences. \\
\hline
\end{tabular}

As people's personalities and cognitive capabilities are diverse, virtual communication might not suit all users. This point added to the challenges that educational leaders encounter. The participant E. H. stated, "People abilities differ and their durability with the use of technology differ as well, and this caused difficulty to accommodate all of these differences".

\section{Discussion}

Over the last decade, organizations have been reforming to accommodate much human interaction through the use of information technology. E-leadership has been penetrating leaders' routines in different sectors. They are expected to 
foster a sense of reality that fosters collaboration and collegiality between the team members. This form of leadership generates different communication which connate new forms of opportunities. In addition to today's explosion in communication technology, leaders have to become increasingly global. E-leadership, especially after experiencing the intensive online work in the COVID-19 crisis, won't be an exception, rather it is going to be a routine. Especially after the Collective awareness of the significance of technology and the altered believes about the efficiency of online communications. Accordingly, leaders must be able to make sense of technology to make the competent use of it.

The occurrence of a crisis is inevitable and unpredictable. Furthermore, leaders perceive these crises in different magnitude. Thus, prior preparations mentally and professionally play a significant role in going through the crisis and reduce change resistance with extracting opportunities out of them. Promoting individual and team adaptability in times of crisis is one of the leaders' responsibilities in leading digitally. Creating the dynamic of effective e-performance among team members requires time to develop; considering the sudden occurrence of crisis which doesn't provide a chance for creating this dynamic, leaders also have to prepare team members to work in different circumstances.

"A crisis is an opportunity riding a dangerous wind." Chinese Proverb. The noted themes are inductively developed by categorizing codes into themes. Many opportunities can be extracted from the experience of e-leadership in times of crisis These findings are compatible with what Kouzes and Posner pointed out that with leadership challenges opportunities arise. Intellectual, professional, organizational, and personal aspects are some of these opportunities [17]. No, constrain by geographical existence; thus, the attained knowledge becomes greater. The ability to communicate instantly with a large number of people, and increasing the followers' and learners' satisfaction by providing free restricted time for communication. This finding was highlighted by Bavel, et al., and Ulmer when they pointed to the importance of and the sensitivity of communication in times of crisis $[5 ; 33]$. The opportunity of forming a multi-functional team and cherry-pick the talent that enhances organizational performance. In addition, to varieties of the skills that are developed out of leading online in crisis. The pandemic also has created leadership opportunities for various groups of people such as students, families, workers by reinforcing self-discipline to perform allocated tasks.

A crisis is a negative event, yet maintaining a positive culture, represented by leaders, is vital for the survival and the strength of the organization. Discussing the challenges that were encountered by the educational leader in leading digitally in crisis makes leadership a complicated task, yet the challenges are manageable and the benefit of e-leadership exceeds the difficulties. The internal and external challenges that educational leaders have faced can be managed by the strategies which are presented by Mazánek and Kielkowski [26; 19]. To clarify, "Calmness in the eye of the storm" as
Lucy articulated it [24]. Besides building trust and practicing effective leadership before and during crisis alleviates the challenge. This is also compatible with Jones and Chauhan's study in which effective leadership in crisis required positive attitudes and calm conduct [18]. With regard to the challenge of cultural differences in accepting online communication, Brake suggested using practical guidelines for effective e-leadership in this matter by increasing cultural intelligence [7]. Unprepared leaders can affect negatively broader audiences; thus, prior preparation to improve skills and adaptability with unpredicted changes or crisis become essentials.

\section{Recommendation}

The findings of this study offer important insight into both the theory and practice for both current and prospective policymakers and educational leaders. They contain several important recommendations that policymakers and educational leaders can consider.

The crisis of coronavirus pandemic will flourish e-learning after experiencing it; hence, educational leaders are expected to reframe their practices to provide an effective leadership style for leading electronically.

Educational leaders have to expand the use of technology in learning and communication and reinforce the effective use of it.

Some people are reluctant to use technology in working or learning. Therefore, as people's behavior is influenced by social norms, creating a culture to adopt more effectively to the use of technology is essential.

Creating a technical alternative to provide education in any circumstances.

Allocating finance for an educational alternative in crisis.

The occurrence of crisis is hard to predict; therefore, prior preparation professionally and mentally to adapting to change is needed for leaders and learners.

New and adaptive skills also are needed to accommodate different types of leadership for leading electronically.

Creating a mutual vision to prevent misunderstanding which might cause by the lack of physical communications. Developing a program/system for responding to an outbreak.

Further multidisciplinary research is needed to confront crises effectively.

Conducting comparative research to benefit from international experiences of dealing with the crisis in the educational sector.

Training leaders and urge them to maintain a work-life balance- and helping others to maintain it.

\section{Conclusion}

The study was conducted to investigate e-leadership in crisis by acquiring data from educational leaders in the crisis of COVID-19. Human interaction in organizations has been significantly mediated by information technology. Leaders are expected to influence and motivate effective performance in a virtual context. Accordingly, they have to be equipped with sufficient preparation to lead effectively especially in times of 
crisis. COVID-19 pandemic has forced educational leaders to lead virtually which showed leaders opportunities and challenges. The opportunities fall under intellectual, professional, organizational, and personal aspects. Whereas, the challenges fall under internal and external management challenges. However, these opportunities and challenges are assets for developing the educational sector and move it into a more advanced digital world with minimizing challenges.

\section{Acknowledgements}

I would like to express my sincere gratitude for University of Jeddah for supporting researchers, and for all the participants in this study.

\section{References}

[1] Akram, M. \& Khan, Ayaz. (2020). Exploring E-Leadership of Principals: Increasing School Effectiveness by Learning Management System. Journal of Education \& Social Sciences, $8 \quad(1)$ 15-30 https://doi-org.sdl.idm.oclc.org/10.20547/jess0812008102

[2] Avolio, B. J., \& Kahai, S. S. (2003). Adding the "E" to E-Leadership: How it may impact your leadership. Organizational dynamics.

[3] Avolio, B., Kahai, S. \& Dodge, G.E. (2000). E-leadership: Implications for Theory, Research, and Practice. Leadership Quarterly, 11 (4), pp. 615-668. Available at: http://ac.els-cdn.com/S104898430000062X/1-s2.0S10489843 0000062X-main.pdf? tid=cf709690-f0d9-11e5-aa49

[4] Avolio, et al. (2014). E-leadership: Re-examining transformations in leadership source and transmission. The Leadership Quarterly, 25, pp. 105-131.

[5] Bavel, J. J. V., Baicker, K., Boggio, P. S. et al. (2020). Using social and behavioural science to support COVID-19 pandemic response. Nat Hum Behav (2020). https://doi.org/10.1038/s41562-020-0884-z.

[6] Bhaduri, R. (2019). Leveraging culture and leadership in crisis management. European Journal of Training and Development.

[7] Brake, T. (2006). Leading global virtual teams. Industrial and Commercial Training, 38 (3).

[8] Charmaz, K. (2014). Constructing grounded theory. London, United Kingdom: SAGE.

[9] Creswell, J. (2013). Qualitative inquiry and research design: Choosing among five approaches. Thousand Oaks, CA: Sage.

[10] Darics, E. (2020). E-Leadership or "How to Be Boss in Instant Messaging?" The Role of Nonverbal Communication. International Journal of Business Communication, 57 (1), 3-29. https://doi-org.sdl.idm.oclc.org/10.1177/2329488416685068

[11] DasGupta, P. (2011). Literature review: e-Leadership. Emerging Leadership Journeys, 4 (1), 1-36.

[12] Denzin, N. K., \& Lincoln, Y. S. (Eds.). (2011). The Sage handbook of qualitative research. Thousand Oaks, CA: Sage Publication.
[13] English, F. W. (2006). Encyclopedia of educational leadership and administration. Thousand Oaks, CA: Sage.

[14] English, F. (2008). The art of leadership: Balancing performance and accountability. Thousand Oaks, CA: Sage Publication.

[15] Garcia, I. (2020). e-Leadership: A Bibliometric Analysis. International Journal of Advanced Corporate Learning, 13 (1), $19-34$.

https://doi-org.sdl.idm.oclc.org/10.3991/ijac.v13i1.12341

[16] Gates, B. (2020). Responding to Covid-19-a once-in-a-century pandemic?. New England Journal of Medicine, 382 (18), 1677-1679.

[17] Jones, M., Massiah, P., \& Sounderraj, M. (2017). E-Leadership: Operationalising an Empirical Research Agenda for Future Theoretical Generation and Assessment. Proceedings of the International Conference on Intellectual Capital, Knowledge Management \& Organizational Learning, 201-209.

[18] Jones, S. \& Chauhan, P. (2019). Leadership in Crisis- Insight from India and Abroad. Effective Executive, 22 (3), 7.

[19] Kielkowski, R. (2013). Leadership During Crisis. Journal of $\begin{array}{llll}\text { Leadership } & \text { Studies, } & 7 & \text { (3), }\end{array}$ https://doi-org.sdl.idm.oclc.org/10.1002/jls.21300

[20] King, G. (2002). Crisis Management \& Team Effectiveness: A Closer Examination. Journal of Business Ethics, 41 (3), pp. 235-249.

[21] Kouzes, J. M., \& Posner, B. Z. (2017). The leadership challenge: how to make extraordinary things happen in organizations. Sixth edition. Hoboken, New Jersey: John Wiley \& Sons, Inc.

[22] Kraft, M. H. G. (2019). How to lead with digital media effectively? A literature-based analysis of media in an E-leadership context. Journal of Economic Development, Environment \& People, 8 (4), 42-53. https://doi-org.sdl.idm.oclc.org/10.26458/jedep.v8i4.639

[23] Loske, D., (2013). Methodological thinking: Basic principles of social science research. Thousand Oaks, CA: Sage Publications.

[24] Lucy, J. (2020). Leadership \& Crisis Management for the Ages. Electrical Wholesaling, 101 (4), 16-23.

[25] Malhotra, A., Majchrzak, A., \& Rosen, B. (2007). Leading virtual teams. Academy Of Management Perspective, 21, 60-70.

[26] Mazánek, L. (2015). Leadership during Crisis: Threat Identification and Solution Proposal. Trends: Economics \& Management / Trendy: Ekonomiky a Managementu, 9 (24), 6170. Oaks, CA: Sage.

[27] Meriam, S. B. (2002). Assessing and evaluating qualitative research. In S. B. Merriam \& Associates (Eds.), Qualitative research in practice: Examples for discussion and analysis (pp. 18-36). San Francisco, CA: Jossey-Bass.

[28] Pal, L. (2014). Beyond policy analysis: Public issue management in turbulent times (5th ed.). Toronto, Canada: Thompson/Nelson.

[29] Preedy, M., Bennett, N., \& Wise, C. (2012). Educational leadership: Context, strategy and collaboration. London, United Kingdom: SAGE. 
[30] Probert, J., \& James, K. (2011). Leadership development: Crisis, opportunities and the leadership concept. Leadership, 7 (2), 137-150

[31] Sathithada, K., \& Niramitchainont, P. (2019). Scenarios of a Sustainable E-leadership for Thai Higher Educational Institution Leaders in 2027. Discourse \& Communication for Sustainable Education, $10 \quad$ (1), 81-90. https://doi-org.sdl.idm.oclc.org/10.2478/dcse-2019-0007

[32] Smith, L., \& Abouammoh, A. (2013). Higher education in Saudi Arabia achievements, challenges and opportunities. New York, NY: Springer.

[33] Ulmer, R. R. (2012). Increasing the impact of thought leadership in crisis communication. Management Communication Quarterly, 26 (4), 523-542.
[34] World Health Organization. Coronavirus. Retrieved from https://www.who.int/health-topics/coronavirus\#tab=tab_1

[35] Zaccaro, S. J., \& Bader, P. (2003). E-leadership and the challenges of leading e-teams: Minimizing the bad and maximizing the good. Organizational dynamics.

\section{Biography}

Faiza Gonaim is an associate professor in the Department of Administration and Fundamentals of Education at University of Jeddah, and the chair of the department. Her research interest is in educational leadership and in approaches that uplift people and unleash their potential in fulfilling their personal and organizational goals. 